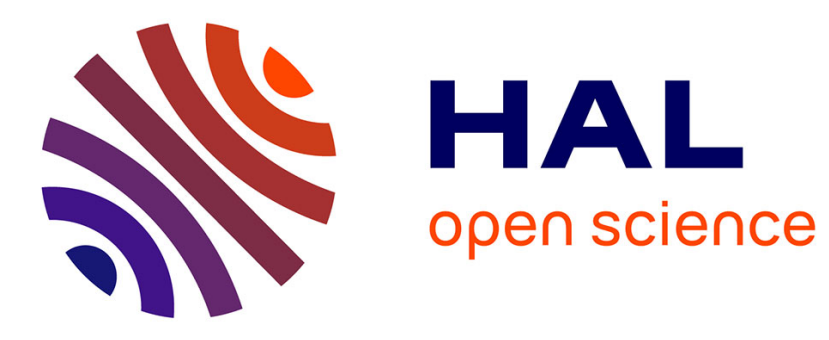

\title{
On the reconstruction of residual stresses after matter removal in rods
}

\author{
Andrei Constantinescu, Abdelbacet Oueslati
}

\section{To cite this version:}

Andrei Constantinescu, Abdelbacet Oueslati. On the reconstruction of residual stresses after matter removal in rods. Comptes rendus de l'Académie des sciences. Série IIb, Mécanique, 2008, 336 (1-2), pp.66-79. 10.1016/j.crme.2007.10.016 . hal-00346668

\section{HAL Id: hal-00346668 \\ https://hal.science/hal-00346668}

Submitted on 12 May 2020

HAL is a multi-disciplinary open access archive for the deposit and dissemination of scientific research documents, whether they are published or not. The documents may come from teaching and research institutions in France or abroad, or from public or private research centers.
L'archive ouverte pluridisciplinaire HAL, est destinée au dépôt et à la diffusion de documents scientifiques de niveau recherche, publiés ou non, émanant des établissements d'enseignement et de recherche français ou étrangers, des laboratoires publics ou privés.

\section{(c)(1)}

Distributed under a Creative Commons Attribution| 4.0 International License 


\title{
On the reconstruction of residual stresses after matter removal in rods
}

\author{
Andrei Constantinescu $^{\mathrm{a}, *}$, Abdelbacet Oueslati ${ }^{\mathrm{b}}$ \\ a Laboratoire de mécanique des solides - CNRS UMR 7649, École polytechnique, 91128 Palaiseau cedex, France \\ ${ }^{\mathrm{b}}$ Laboratoire de mécanique de Lille - CNRS UMR 8107, Université de sciences et technologies de Lille, cité scientifique, \\ boulevard Paul-Langevin, 59655 Villeneuve d'Ascq cedex, France
}

\begin{abstract}
This article discusses the reconstruction methods for the inversion of the residual stresses in rods under the Navier-Bernoulli or the Vlassov (containing warping) kinematic conditions. The proposed framework permits us to express the residual stresses in terms of the Airy stress potential expressed as the solution of an ordinary differential equation. The particular case of the NavierBernoulli kinematics leads to a closed-form solution.
\end{abstract}

\section{Résumé}

Sur la reconstruction de champs des contraintes résiduelles dans les barres après enlèvement de la matière. Cet article présente les méthodes de reconstruction des contraintes résiduelles dans des poutres en considérant des hypothèses cinématiques de type Navier-Bernoulli ou Vlassov (avec gauchissment). La technique proposée permet d'exprimer les contraintes résiduelles à partir d'une fonction d'Airy, solution d'une équation différentielle ordinaire. La cinématique de Navier-Bernoulli conduit à une solution explicite de ce problème.

Keywords: Solids and structures; Residual stresses; Rod theory; Warping

Mots-clés : Solides et structures ; Contraintes résiduelles ; Poutres ; Gauchissement

\section{Version française abrégée}

La détermination des contraintes résiduelles dans les structures est un sujet de grande importance puisque leur présence affecte considérablement la résistance à la fatigue et à la rupture des pièces mécaniques.

Deux classes de méthodes d'identification peuvent être utilisées : les méthodes non destructives (mesures par diffraction des rayons X, par ultrasons, etc.) et destructives (enlèvement de la matière, perçage de trou, etc.). Ces dernières provoquent un relâchement de contraintes (et de déformation) conduisant ainsi à un problème inverse de reconstruction de champ de contraintes initial à partir de mesures effectuées sur la frontière du solide.

\footnotetext{
* Corresponding author.

E-mail addresses: andrei.constantinescu@1ms.polytechnique.fr (A. Constantinescu), Abdelbacet.Oueslati@univ-lille1.fr (A. Oueslati).
} 
Dans cet article, nous présentons les méthodes de reconstruction des contraintes résiduelles après enlèvement de la matière dans le cas des barres élancées de section rectangulaire. L'hypothèse principale adoptée est celle d'une relaxation purement élastique des contraintes après enlèvement successif de couches d'épaisseurs $h$. Cette hypothèse permet de ramener la résolution du problème de reconstruction à l'étude de trois problèmes élastiques distincts (voir Fig. 2) : le premier problème $\mathbf{P 1}$ est celui de l'équilibre de la poutre $\Omega$ libre de charges extérieures et contenant le champ de contraintes résiduelles inconnu $\boldsymbol{\sigma}$. Pour le deuxième problème noté $\mathbf{P 2}$, le solide est constitué par la barre $\Omega_{h}$ obtenue à partir de $\Omega$ après enlèvement d'une couche d'épaisseur $h$, c'est sur cette configuration que la mesures des contraintes par diffraction des rayons X est effectuée sur la face supérieure. Enfin, $\mathbf{P 3}$ est le problème élastique auxiliaire traduisant l'équlibre de la barre $\Omega_{h}$, soumise aux tractions surfaciques $\left(-\sigma \cdot \mathbf{e}_{z}\right)$ sur sa face supérieure ( $\sigma$ étant le champ de contraintes résiduelles à identifier). L'application du principe de superposition à ces trois problèmes conduit à la relation (3).

Il est impératif de noter que le problème de reconstruction possède une solution explicite si le système (2) et (3), ainsi que le problème auxiliaire ont des solutions explicites. Rappelons que pour le cas d'un demi-espace élastique, la solution du problème auxiliare $\mathbf{P 3}$ est fournie d'une manière explicite par la solution de Boussinesq-Cerutti et le système (2) est résolu après application de la transformée de Fourrier [2].

Pour le cas des barres de dimensions finies, une solution explicite analogue à celle de Boussinesq-Cerutti est difficille à établir. L'idée est de résoudre le problème auxiliaire dans le cadre de la théorie des poutres de NavierBernoulli puis celle de Vlasov et d'introduire le potentiel de contraintes d'Airy (13) pour résoudre les Éqs. (2) et (3).

Des formules intégrales explicites (16) pour la reconstruction de champ de contraintes résiduelles sont obtenues pour la cinématique de Navier-Bernoulli. Elles constituent une généralisation des formules de Morre et Avans [3] établies pour les barres (plaques) de longueurs infinies.

\section{Introduction}

Identification of residual stresses is an important task in many engineering fields such as fatigue or fracture mechanics, where their presence can significantly increase or decrease the apparent strength of mechanical components.

The problem of stress redistribution after matter removal has instructively been illustrated by Prof. H.D. Bui who has sectioned a commercial aluminium rod. The two parts were highly deformed after the cut due to the presence of residual stresses as displayed in Fig. 1. The present article is intended to bring a modest contribution to this problem.

Accurate measurements of residual stresses are performed by non-destructive techniques X-ray diffraction measurements at the surface of structures or by destructive methods: hole-drilling, layer removal, etc., if one wants to explore the residual stress field in depth of the solid. This relies essentially on measuring the stress redistribution produced by matter removal on a body containing residual stresses produced by an incompatible initial thermal or plastic strain distribution. In such a case, one supposes that matter removal leads to an elastic redistribution of stresses and

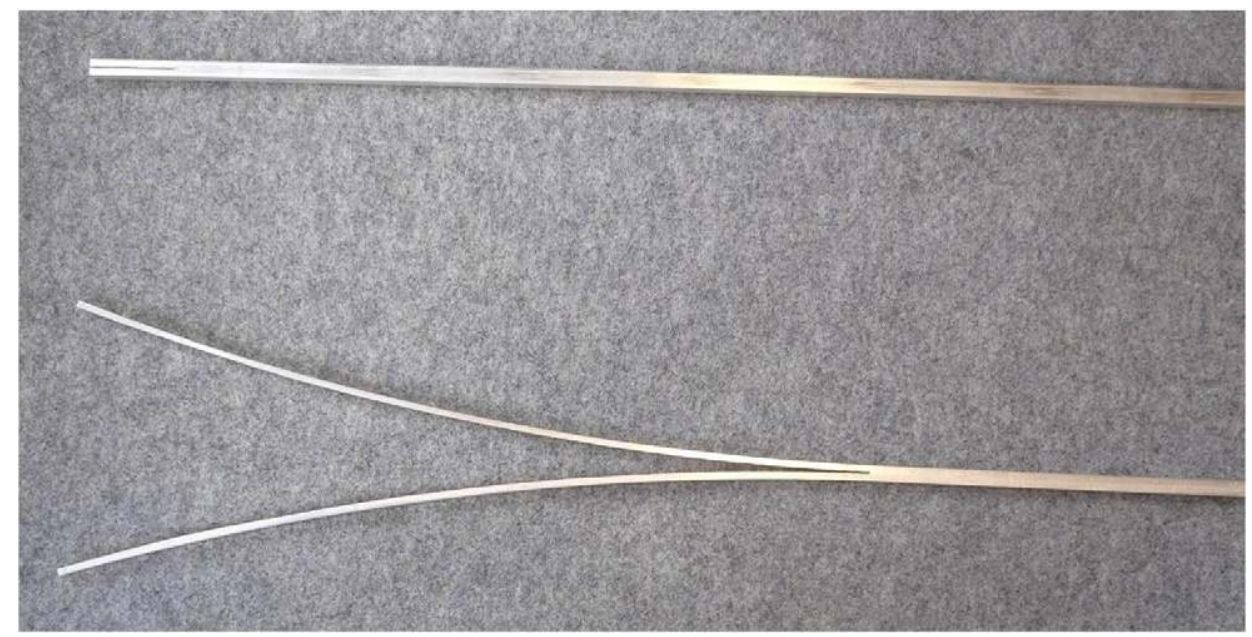

Fig. 1. Deformation of an aluminium rod after cutting, due to the presence of residual stresses, an experiment proposed by Prof. H.D. Bui. 
measures generally the strain or the stresses on the boundary after the matter removal by means of X-ray diffraction or by neutron diffraction. This technique conducts therefore to an inverse reconstruction problem of the initial residual stress before mater removal from measurements performed after matter removal.

The study of the matter removal problem can be related to an evolution problem and uniqueness of its solution has already been proven in [1] for bodies of arbitrary shape.

For a practical purpose, Moore and Evans [3] proposed for the matter removal technique, in their pioneering paper, a series of exact integral formulas for the reconstruction of residual stress field in the following cases: (a) solid rod and hollow cylinder (tube) with rotationally symmetric stresses independent of the axial coordinate $z$; (b) flat plate with stresses varying along the thickness of the plate. These formulas apply only in cases where the residual stress field depends on just one spatial variable (corresponding to depth). However, many configurations arise in applications where the stress fields are varying along the surface of the body. Explicit formulas for such cases have been obtained only in the following cases: (i) hollow cylinder (tube) with rotationally symmetric stresses and varying along the $z$-coordinate (exact infinite series expression given by Nishimura [4]); (ii) two and three dimensional half-spaces with stresses depending of all spatial variables (closed-form expression obtained by Ballard and Constantinescu [2]). The main difficulties in finding closed-form solutions of this problem for bodies of different shapes can be related to: (i) the existence of a fundamental solution of Boussinesq-Cerruti type of the elastic problem for the body under discussion; and (ii) the integration of the evolution problem defined previously.

This article proposes to fill the gap between axially homogenous stresses in infinite bars (plates) and varying stresses in a semi-infinite domains by discussing the case of slender finite dimensional rectangular solids within the framework of rod theory.

Rod theories, can be interpreted in terms of power series developments of the three dimensional elastic solution in term of a small parameter, i.e. the diameter of the cross-section of the rod. Expanding the three dimensional 'exact' elastic solution up to different orders one can obtain explicit successive approximations including bending, torsion, warping and Poisson effects of the beam under study. A detailed mathematical proof can be found in Trabucho et al. [5].

The asymptotic expansions of the three dimensional displacement and stress field are given in the following power series of odd power terms:

$$
\boldsymbol{u}(e)=\boldsymbol{u}^{\mathbf{0}}+e^{2} \boldsymbol{u}^{2}+e^{4} \boldsymbol{u}^{4}+\text { h.o.t., } \quad \boldsymbol{\sigma}(e)=\boldsymbol{\sigma}^{\mathbf{0}}+e^{2} \boldsymbol{\sigma}^{2}+e^{4} \boldsymbol{\sigma}^{4}+\text { h.o.t. }
$$

where h.o.t. are higher order terms and $e$ is the diameter of the cross-section.

The main conclusion of Trabusho et al. [5] is that without further assumptions on the loads or geometry, the zero order term of the asymptotic expansion i.e. $\left(\boldsymbol{u}^{\mathbf{0}}, \boldsymbol{\sigma}^{\mathbf{0}}\right)$ coincides with the Navier-Bernoulli model and the second order terms $\left(\boldsymbol{u}^{\mathbf{0}}, \boldsymbol{\sigma}^{\mathbf{0}}\right)+e^{2}\left(\boldsymbol{u}^{\mathbf{2}}, \boldsymbol{\sigma}^{\mathbf{2}}\right)$ permits the justification of the Saint-Venant model for simple torsion, Timoshenko's bending equations and Vlasov's rod theory [6].

This paper discusses the reconstruction formulas for the inversion of the residual stresses in rods under the NavierBernoulli or the Vlassov (containing warping) kinematic conditions. The proposed framework permits one to express the residual in term of the Airy stress potential expressed as the solution of an ordinary differential equation. The particular case of the Navier-Bernoulli kinematics conducts to a closed-form solution.

\section{The reconstruction problem}

Let $\Omega$ be a free rod of rectangular section and dimensions $a \times b \times c$ referred to a Cartesian coordinate system $(x, y, z)$ (see Fig. 2). The neutral fibre coincides with $(O x)$ and $(O y, O z)$ are principle axes of inertia of the cross-
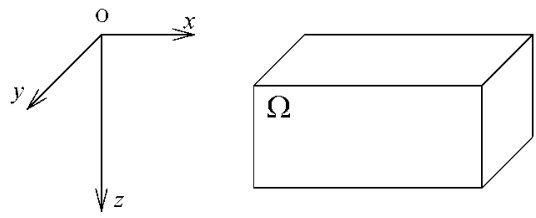

$\sigma$

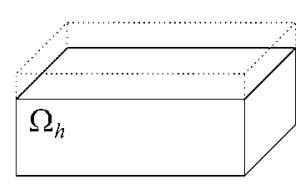

$\sigma^{\mathrm{m}}$

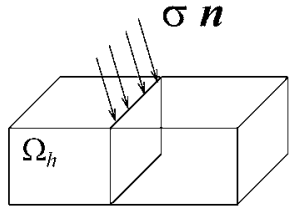

$\sigma^{h}$

Fig. 2. The three problems for the rod. 
section. This simplifying assumptions are made for the clearness of the presentation and do not represent a restriction to the generality of the setting of this problem.

From $\Omega$, layers of constant thickness $h$ are removed continuously, leaving for each $h$ a new rod $\Omega_{h}$. During the matter removal procedure we assume that:

H1 The relaxation of the body due to matter removal is elastic.

In order to define a method of solution, as in the three dimensional case, one can split the reconstruction problem into three elastic equilibrium problems (see Fig. 2):

P1 The equilibrium of the initial solid $\Omega$ with the unknown distribution plastic strain field $\varepsilon^{p}$ creating residual stress field to be reconstructed. This field $\sigma$ satisfies the balance equations:

$$
\operatorname{div} \sigma=0, \quad\left\{\begin{array}{l}
\sigma_{x x, x}+\sigma_{x z, z}=0 \\
\sigma_{x z, x}+\sigma_{z z, z}=0
\end{array}\right.
$$

P2 The equilibrium of $\Omega_{h}$, the rod after matter removal containing the undisturbed plastic strain field $\boldsymbol{\varepsilon}^{p}$. This is the configuration on which surface stress measurements are performed by means of X-ray diffraction method.

P3 The auxiliary problem defined on $\Omega_{h}$ is a purely elastic problem, with a stress distribution $\sigma^{h}$ created by surface tractions on the upper surface given by:

$$
\boldsymbol{t}=-\boldsymbol{\sigma}(x, h) \cdot \boldsymbol{e}_{z}=-\sigma_{x z}(x, h) \boldsymbol{e}_{x}-\sigma_{z z}(x, h) \boldsymbol{e}_{z}
$$

where $\sigma$ is the residual stress field of problem P1. In order to recall later this dependence we shall use the following notation:

$$
\boldsymbol{\sigma}^{h}(x, z)=G[\boldsymbol{t}](x, z)=G\left[\sigma_{x z}, \sigma_{z z}\right](x, z), \quad z \in[h, c]
$$

Under the $\mathbf{H 1}$ assumption of an elastic redistribution of stresses, one can now apply the superposition principle and as a consequence the following equality between the components of the residual stress components $\sigma_{x x}$ of the three problems can be established:

$$
\sigma_{x x}(x, h)=\sigma_{x x}^{m}(x, h)+\sigma_{x x}^{h}(x, h)=\sigma_{x x}^{m}(x, h)+G\left[\sigma_{z x}, \sigma_{z z}\right](x, h)
$$

This implicit relation between the components of the residual stress and the measurement $\sigma_{x x}^{m}$ represents together with the balance equation (2), a system of equations for the unknown components of the residual stress.

The problem has a closed-form solution provided both the expression $G\left[\sigma_{z x}, \sigma_{z z}\right]$ and the system (2) and (3) have a closed form solution.

In the case of the half space problem $G$ was computed using the Boussinesq-Cerutti stress potentials and the system has been transformed into an ordinary differential equation using an integral transform which could be integrated.

In the present case of a slender domain of finite extension, the main idea is to use the expression of $G$ defined in the framework of an imposed rod kinematics for the problem. The balance equation will be eliminated by the use of the Airy stress potential $\Phi$ to express the residual stress $\sigma$.

\section{Reconstruction for the Navier-Bernoulli rod}

Let us consider a rod with the longitudinal axes along its mean fibre. According to the Navier-Bernoulli (LoveKirchhoff) beam theory [6], the rod kinematics is defined by the following form of displacement field:

$$
\boldsymbol{u}=\left(u(x)-w_{, x}(x) z\right) \boldsymbol{e}_{x}+w(x) \boldsymbol{e}_{z}
$$

where $u(x)$ and $w(x)$ are the axial translation and the transversal deflexion of the cross-section respectively. ' ${ }_{,}$' denotes the partial derivative with respect to $x$. The angle of rotation of the cross-section is $-w_{, x}(x)$ and signifies that the cross-section remains orthogonal to the mean fibre during the deformation of the rod. Moreover, the cross-section is rigid in its plane. 


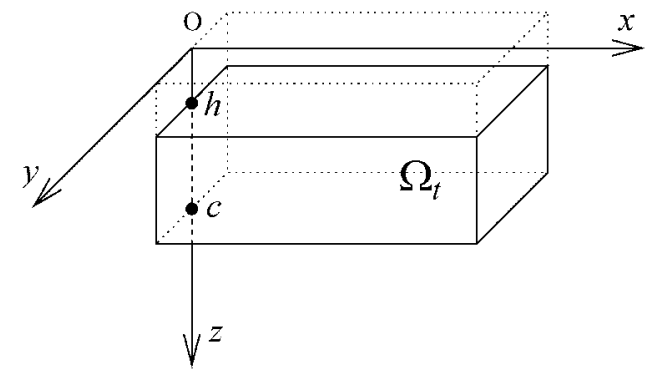

Fig. 3. The coordinates for the rod after removal of a layer of thickness $h$.

The three dimensional strain of the rod is related with the displacement field under the small strain assumption by:

$$
\boldsymbol{\varepsilon}=\varepsilon_{x x} \boldsymbol{e}_{x} \otimes \boldsymbol{e}_{x}=\left(u_{, x}-w_{, x x}\right) \boldsymbol{e}_{x} \otimes \boldsymbol{e}_{x}
$$

The stress field is expressed for a linear elastic isotropic material as:

$$
\boldsymbol{\sigma}=\varepsilon_{x x}\left((\lambda+2 \mu) \boldsymbol{e}_{x} \otimes \boldsymbol{e}_{x}+\lambda\left(\boldsymbol{e}_{y} \otimes \boldsymbol{e}_{y}+\boldsymbol{e}_{z} \otimes \boldsymbol{e}_{z}\right)\right)
$$

where $\lambda$ and $\mu$ are the Lamé moduli. One can remark that the kinematic assumption neglects the shear stress, which does not imply that the shear forces do vanish. The generalized stresses for the beam theory are related to the three dimensional stress components and to displacement field through:

$$
N(x)=\int_{S} \sigma_{x x}(x, z) \mathrm{d} s=E S u_{, x}(x), \quad M_{y}(x)=\int_{S} z \sigma_{x x}(x, z) \mathrm{d} s=-E I w_{, x x}(x)
$$

where $N(x)$ and $M_{y}(x)$ stand for the resultant force and the resultant bending moment of the section of coordinate $x$. $S$ denotes the area of the cross-section and $I=\int_{S} z^{2} \mathrm{~d} S$ is the moment of inertia.

The auxiliary problem $\Omega_{h}(0<h \leqslant c$, see Fig. 3) expresses the of equilibrium the following forces and stresses:

$$
\left\{\begin{array}{l}
N_{, x}^{h}+f_{x}^{h}=0 \\
M_{, x x}^{h}+m_{y, x}^{h}+f_{z}^{h}=0
\end{array}\right.
$$

The exterior loads are related to the components of residual stress $\sigma$ by the following expressions:

$$
\begin{aligned}
& \boldsymbol{f}^{h}(x)=-b \sigma_{x z}(x, h) \boldsymbol{e}_{x}-b \sigma_{z z}(x, h) \boldsymbol{e}_{z} \\
& \boldsymbol{m}_{y}^{h}(x)=b \sigma_{x z}(x, h) \frac{(c-h)}{2} \boldsymbol{e}_{y}
\end{aligned}
$$

Supposing that the section $x=0$ is traction free, the solution of the auxiliary problem can be defined as:

$$
\sigma_{x x}^{h}(x, z)=\frac{N^{h}(x)}{b(c-h)}+\frac{12 M_{y}^{h}(x)}{b(c-h)^{3}}\left(z-\frac{c+h}{2}\right)
$$

Using the previous formulae on the upper surface $z=h$ (see Fig. 3) in the system of Eqs. (2) and (3) one obtains:

$$
\left\{\begin{array}{l}
\sigma_{x x}(x, z)=\sigma_{x x}^{m}(x, z)+\frac{4}{c-z} \int_{0}^{x} \sigma_{x z}(u, z) \mathrm{d} u-\frac{6}{(c-z)^{2}} \int_{0}^{x} \int_{0}^{u}\left(\sigma_{z z}\left(u^{\prime}, z\right) \mathrm{d} u^{\prime}\right) \mathrm{d} u \\
\frac{\partial \sigma_{x x}}{\partial x}+\frac{\partial \sigma_{x z}}{\partial z}=0 \\
\frac{\partial \sigma_{x z}}{\partial x}+\frac{\partial \sigma_{z z}}{\partial z}=0
\end{array}\right.
$$

where $\sigma_{x x}^{m}$ is the stress supplied by the X-ray measurements and $\sigma$ are the residual stresses to be reconstructed.

In order to solve this system, we shall introduce the Airy function $\Phi(x, z)$ :

$$
\sigma_{x x}=\frac{\partial^{2} \Phi}{\partial z^{2}}, \quad \sigma_{x z}=-\frac{\partial^{2} \Phi}{\partial x \partial z}, \quad \sigma_{z z}=\frac{\partial^{2} \Phi}{\partial x^{2}}
$$


Therefore equilibrium equations in (12) are automatically satisfied and the system reduces to following ordinary differential equation:

$$
\frac{\partial^{2} \Phi}{\partial Z^{2}}-\frac{4}{Z} \frac{\partial \Phi}{\partial Z}+\frac{6}{Z^{2}} \Phi=\sigma_{x x}^{m}
$$

where $Z=c-z$. The conditions $\Phi(0, z)=0, \frac{\partial \Phi}{\partial z}(0, z)=0$ and $\frac{\partial \Phi}{\partial x}(0, z)=0$ can be obtained by integrating the boundary conditions for the stress field.

Eq. (14) is a second order Euler equation and the general solution has the form:

$$
\Phi(x, Z)=A(x) Z^{2}+B(x) Z^{3}+Z^{2} \int_{Z}^{h} \frac{\sigma_{x x}^{m}\left(x, z^{\prime}\right)}{z^{\prime}} \mathrm{d} z^{\prime}-Z^{3} \int_{Z}^{h} \frac{\sigma_{x x}^{m}\left(x, z^{\prime}\right)}{z^{\prime 2}} \mathrm{~d} z^{\prime}
$$

Using the boundary conditions $\sigma_{x z}=0$ and $\sigma_{z z}=0$ on the upper surface $Z=c$ as well as $\sigma_{x x}=0$ on the end sections one obtains $A(x)=B(x)=0$. A straightforward computations gives now the expression of the initial residual stresses as a function of the measured surface stress:

$$
\left\{\begin{array}{l}
\sigma_{x x}(x, z)=\sigma_{x x}^{m}(x, z)+2 \int_{0}^{z} \frac{\sigma_{x x}^{m}\left(x, z^{\prime}\right)}{c-z^{\prime}} \mathrm{d} z^{\prime}-6(c-z) \int_{0}^{z} \frac{\sigma_{x x}^{m}\left(x, z^{\prime}\right)}{\left(c-z^{\prime}\right)^{2}} \mathrm{~d} z^{\prime} \\
\sigma_{x z}(x, z)=2(c-z) \int_{0}^{z} \frac{\sigma_{x x, x}^{m}\left(x, z^{\prime}\right)}{c-z^{\prime}} \mathrm{d} z^{\prime}-3(c-z)^{2} \int_{0}^{z} \frac{\sigma_{x x, x}^{m}\left(x, z^{\prime}\right)}{\left(c-z^{\prime}\right)^{2}} \mathrm{~d} z^{\prime} \\
\sigma_{z z}(x, z)=(c-z)^{2} \int_{0}^{z} \frac{\sigma_{x x, x x}^{m}\left(x, z^{\prime}\right)}{c-z^{\prime}} \mathrm{d} z^{\prime}-(c-z)^{3} \int_{0}^{z} \frac{\sigma_{x x, x x}^{m}\left(x, z^{\prime}\right)}{\left(c-z^{\prime}\right)^{2}} \mathrm{~d} z^{\prime}
\end{array}\right.
$$

A simple inspection of the formulae show that they still hold if the width of the bar depends of the spatial variable $x$.

One can remark that imposing $\sigma_{x x, x}=0$ conducts formally to the original Moore and Evans formulae. The resemblance is only formal because the condition $\sigma_{x x, x}=0$ on a rod of finite length implies that $\sigma_{x x}=0$. Another remark is the apparent contradiction between the Navier-Bernoulli rod kinematics imposing $\sigma_{x z}=0$ and the final solution where $\sigma_{x z} \neq 0$. However, a close inspection shows that this is directly related to the approximation given by the rod kinematics. In order to improve the accuracy in this respect we shall next propose to enrich the rod kinematics.

\section{Reconstruction for the Vlasov rods with warping}

\subsection{General Vlasov rods with warping}

The kinematical enrichment proposed by Vlasov's model in [6] is based on the following form of displacement field:

$$
\boldsymbol{u}=\left(u_{1}(x)+u_{2}(x) z+u_{3}(x) \varphi(z)\right) \boldsymbol{e}_{x}+w(x) \boldsymbol{e}_{z}
$$

where $u_{1}(x), w(x), u_{2}(x)$ and $u_{3}(x)$ are the axial translation, the vertical deflexion, the rotation angle of the crosssection and the warping amplitude, respectively. The function $\varphi$ represents the warping, i.e. out of plane axial displacement of the section. $\varphi$ is supposed to be a known function and satisfies a serie of orthogonality conditions. As an illustration let us recall that warping of rods with an open profile section is necessarily related to the torsion, whereas for full or closed mean profile, warping can results also from bending and tension.

In this theory, the small strain tensor is expressed as:

$$
\boldsymbol{\varepsilon}=\left(\begin{array}{ccc}
\varepsilon_{x x} & 0 & \varepsilon_{x z} \\
0 & 0 & 0 \\
\varepsilon_{x z} & 0 & 0
\end{array}\right)=\left(\begin{array}{ccc}
u_{1, x}+u_{2, x} z+u_{3, x} \varphi & 0 & \frac{1}{2}\left(u_{2}+u_{3} \varphi_{, z}+w_{, x}\right) \\
0 & 0 & 0 \\
\frac{1}{2}\left(u_{2}+u_{3} \varphi_{, z}+w_{, x}\right) & 0 & 0
\end{array}\right)
$$


In linear elasticity, the three dimensional stress, is obtained from Hooke's law:

$$
\sigma=\left(\begin{array}{ccc}
(\lambda+2 \mu) \varepsilon_{x x} & 0 & 2 \mu \varepsilon_{x z} \\
0 & \lambda \varepsilon_{x x} & 0 \\
2 \mu \varepsilon_{x z} & 0 & \lambda \varepsilon_{x x}
\end{array}\right)
$$

The equilibrium equations are established by use of the Principle of Virtual Work and using the orthogonality conditions verified by the warping function $\varphi$ :

$$
|\varphi|_{S}=0 \quad \text { and } \quad|z \varphi|_{S}=0 \quad \text { with }|\cdot|_{S}=\int_{S} \cdot \mathrm{d} s
$$

They are expressed as:

$$
\left\{\begin{array}{l}
E_{1} S u_{1, x x}+f_{x}=0 \\
\mu S u_{2, x}+\mu S w_{, x x}+\mu\left|\varphi_{, z}\right|_{S} u_{3, x}+f_{z}=0 \\
E_{1} I u_{2, x x}-\mu S u_{2}-\mu\left|\varphi_{, z}\right|_{S} u_{3}-\mu S w_{, x}+m_{y}=0 \\
E_{1}\left|\varphi^{2}\right|_{S} u_{3, x x}-\mu\left|\varphi_{, z}\right|_{S} u_{2}-\mu\left|\varphi_{, z}^{2}\right|_{S} u_{3}-\mu\left|\varphi_{, z}\right|_{S} w_{, x}+r_{\varphi}=0
\end{array}\right.
$$

with $E_{1}=\frac{1-v}{(1+v)(1-2 v)} E, E$ the Young modulus and $v$ the Poisson ratio. The external loading is given by the distributed forces and respectively moments:

$$
\boldsymbol{f}=f_{x}(x) \boldsymbol{e}_{x}+f_{z}(x) \boldsymbol{e}_{z}, \quad m_{y}(x)=f_{z}(x) x, \quad r_{\varphi}(x, z)=f_{x}(x) \varphi(z)
$$

The generalized stresses are related to the displacement and the three dimensional stresses by the following equations:

$$
\left\{\begin{array}{l}
N=\int_{S} \sigma_{x x}(x, z) \mathrm{d} s=E S u_{1, x} \\
M_{y}=\int_{S} z \sigma_{x x}(x, z) \mathrm{d} s=E I u_{2, x} \\
B=\int_{S} \varphi(z) \sigma_{x x}(x, z) \mathrm{d} s=E_{1}\left|\varphi^{2}\right|_{S} u_{3, x} \\
T_{\varphi}=\int_{S} \sigma_{x z}(x, z) \varphi_{, z}(z) \mathrm{d} s=\mu\left|\varphi_{, z}\right|_{S}\left(u_{2}+w_{, x}\right)+\mu\left|\varphi_{, z}^{2}\right|_{S} u_{3} \\
T=\int_{S} \sigma_{x z}(x, z) \mathrm{d} s=\mu S u_{2}+\mu\left|\varphi_{, z}\right|_{S} u_{3}+\mu S w_{, x}
\end{array}\right.
$$

where $I=\left|z^{2}\right|_{S}$ and $S=|1|_{S} . N, T$ and $M$ denote the axial force, the shear force and the bending moment respectively, defined as in the Navier-Bernoulli rod theory. $B$ and $T_{\varphi}$ denote the bimoment and the second shear force, respectively.

As for the previous section, one can write the solution of the auxiliary problem for each $\Omega_{h}(0<h \leqslant c)$, within the Vlasov kinematics, given by:

$$
\left\{\begin{array}{l}
N_{, x}^{h}+f_{x}^{h}(x)=0 \\
T_{, x}^{h}+f_{z}^{h}=0 \\
M_{y, x}^{h}+T^{h}+m^{\prime}=0 \\
B_{, x}^{h}-T_{\varphi h}^{h}+r_{\varphi}^{h}=0
\end{array}\right.
$$

This provides the stress solution of the auxiliary problem:

$$
\sigma_{x x}^{h}(x, z)=\frac{N^{h}(x)}{b(c-h)}+\frac{12 M_{y}^{h}(x)}{b(c-h)^{3}}\left(z-\frac{c+h}{2}\right)+\frac{B^{h}(x)}{\left|\varphi_{h}^{2}\right|_{S_{h}}} \varphi_{h}\left(z-\frac{c+h}{2}\right)
$$


It is important to note that the warping functions are now parametrized by the height of the removed layer $h$ and therefore denoted as $\varphi_{h}$.

The system of Eqs. (2) is therefore reduced to the differential equation for the unknown residual stress field:

$$
\left\{\begin{array}{l}
\sigma_{x x}(x, z)=\sigma_{x x}^{m}(x, z)+\frac{4}{c-z} \int_{0}^{x} \sigma_{x z}(u, z) \mathrm{d} u-\frac{6}{(c-z)^{2}} \int_{0}^{x} \int_{0}^{u} \sigma_{z z}\left(u^{\prime}, z\right) \mathrm{d} u^{\prime} \mathrm{d} u+\frac{\varphi_{z}\left(\frac{z-c}{2}\right)}{\left|\varphi_{z}^{2}\right|_{S_{z}}} B^{z}(x) \\
\frac{\partial \sigma_{x x}}{\partial x}+\frac{\partial \sigma_{x z}}{\partial z}=0 \\
\frac{\partial \sigma_{x z}}{\partial x}+\frac{\partial \sigma_{z z}}{\partial z}=0
\end{array}\right.
$$

As before, the expression of the stress field using an Airy potential function $\Phi(x, z)$ eliminates the equilibrium equations in (24) leading to the following expression of the bimoment:

$$
B^{z}(x)=b \int_{z}^{c} \sigma_{x x}\left(x, z^{\prime}\right) \varphi\left(z^{\prime}\right) \mathrm{d} z=b \int_{z}^{c} \frac{\partial^{2} \Phi}{\partial z^{2}}\left(x, z^{\prime}\right) \varphi\left(z^{\prime}\right) \mathrm{d} z^{\prime}
$$

The system (24) becomes:

$$
\frac{\partial^{2} \Phi}{\partial z^{2}}-\frac{4}{c-z} \frac{\partial \Phi}{\partial z}+\frac{6}{(c-z)^{2}} \Phi-b \frac{\varphi_{z}\left(\frac{z-c}{2}\right)}{\left|\varphi_{z}\right| S_{z}} \int_{z}^{c} \frac{\partial^{2} \Phi}{\partial z^{2}}\left(x, z^{\prime}\right) \varphi_{z}\left(z^{\prime}\right) \mathrm{d} z^{\prime}=\sigma_{x x}^{m}(x, c-z)
$$

Using the boundary conditions $\Phi(0, z)=0$ and $\frac{\partial \Phi}{\partial z}(0, z)=0$, one finally obtains:

$$
\frac{\partial^{2} \Phi}{\partial z^{2}}-\frac{4}{c-z} \frac{\partial \Phi}{\partial z}+\frac{6}{(c-z)^{2}} \Phi-b \frac{\varphi_{z}\left(\frac{z-c}{2}\right)}{\left.\mid \varphi_{z}^{2}\right)\left.\right|_{S_{h}}} \int_{z}^{c} \Phi\left(x, z^{\prime}\right) \frac{\partial^{2} \varphi\left(z^{\prime}\right)}{\partial z^{2}} \mathrm{~d} z^{\prime}=\sigma_{x x}^{m}(x, z) .
$$

Unfortunately, this integral-differential equation does not have a closed-form solution for a general form of the warping function $\varphi$. From the practical point of view, one has to choose first the warping function $\varphi$ adapted to the rod profile and integrate afterwards Eq. (26) symbolically or numerically.

\subsection{Vlasov rod with warping and Navier-Bernoulli assumption}

An interesting result is obtained for the particular case of Vlasov kinematics with wrapping combined with the Navier-Bernoulli assumption of orthogonality of the cross-section and the mean fibre during deformation. This condition is expressed as: $u_{2}(x)=-w_{, x}(x)$, and the general form of the displacement is:

$$
\boldsymbol{u}=\left(u_{1}(x)-w_{, x}(x) z+u_{3}(x) \varphi(z)\right) \boldsymbol{e}_{x}+w(x) \boldsymbol{e}_{z}
$$

The second shear force $T_{\varphi}$ is in this case related to the shear force $T$ by:

$$
T_{\varphi}=\frac{\left|\varphi_{, z}^{2}\right|_{S}}{\left|\varphi_{, z}\right| S} T
$$

The expression of generalized stresses is reduced to:

$$
\left\{\begin{array}{l}
N(x)=b \int_{0}^{x} \sigma_{x z}(u, h) \mathrm{d} u \\
M(x)=b \int_{0}^{x} \int_{0}^{u} \sigma_{z z}\left(u^{\prime}, h\right) \mathrm{d} u^{\prime} \mathrm{d} u-b \frac{c-h}{2} \int_{0}^{x} \sigma_{x z}(u, h) \mathrm{d} u \\
B(x)=-b \frac{\left|\varphi_{, z}^{2}\right|}{\left|\varphi_{, z}\right|} \int_{0}^{x} \int_{0}^{u} \sigma_{z z}\left(u^{\prime}, h\right) \mathrm{d} u^{\prime} \mathrm{d} u+b \varphi\left(\frac{h-c}{2}\right) \int_{0}^{x} \sigma_{x z}(u, h) \mathrm{d} u
\end{array}\right.
$$


Under the kinematic assumptions of this theory, the expression of the solution of the auxiliary problem $\sigma_{x x}^{h}$ becomes:

$$
\sigma_{x x}^{h}(x, h)=\alpha^{h} \int_{0}^{x} \sigma_{x z}(u, h) \mathrm{d} u-\beta^{h} \int_{0}^{x} \int_{0}^{u} \sigma_{z z}\left(u^{\prime}, h\right) \mathrm{d} u^{\prime} \mathrm{d} u
$$

where

$$
\alpha^{h}=\frac{4}{c-h}+\frac{b}{\left|\varphi_{z}^{2}\right| S_{h}} \varphi_{h}^{2}\left(\frac{h-c}{2}\right), \quad \beta^{h}=\frac{6}{(c-h)^{2}}+b \varphi_{h}\left(\frac{h-c}{2}\right) \frac{\left|\varphi_{h_{, z}}^{2}\right|_{S_{h}}}{\left|\varphi_{h_{, z}}\right|_{S_{h}}}
$$

The system (24) is now only reduced to:

$$
\left\{\begin{array}{l}
\sigma_{x x}(x, z)=\sigma_{x x}^{m}(x, z)+\alpha^{z} \int_{0}^{x} \sigma_{x z}(u, z) \mathrm{d} u-\beta^{z} \int_{0}^{x} \int_{0}^{u}\left(\sigma_{z z}\left(u^{\prime}, z\right) \mathrm{d} u^{\prime}\right) \mathrm{d} u \\
\frac{\partial \sigma_{x x}}{\partial x}+\frac{\partial \sigma_{x z}}{\partial z}=0 \\
\frac{\partial \sigma_{x z}}{\partial x}+\frac{\partial \sigma_{z z}}{\partial z}=0
\end{array}\right.
$$

Again introducing Airy $\Phi(x, z)$ stress potential we obtain the following differential equation:

$$
\frac{\partial^{2} \Phi}{\partial z^{2}}-\alpha^{z} \frac{\partial \Phi}{\partial z}+\beta^{z} \Phi=\sigma_{x x}^{m}
$$

with the boundary conditions $\Phi(0, z)=0, \frac{\partial \Phi}{\partial z}(0, z)=0$ and $\frac{\partial \Phi}{\partial x}(0, z)=0$.

This second order partial differential equation can generally be solved in a symbolic way, provided the expression of the functions $\alpha^{z}$ and $\beta^{z}$ are explicit. This can be achieved by a particular choice of the warping function $\varphi$.

\section{Conclusion}

The aim of this article was to investigate the problem of reconstructing residual stress from measurements after matter removal in slender bodies. The technique used was based on the superposition principle applied to the initial domain and the domain after matter removal. For all studies rod kinematics the residual stresses were directly related to the solution of an ordinary differential equation on vertical lines of the cross-sections of the rod.

An interesting point is to compare the information necessary to compute the initial residual stress field in an inner point of the body:

- for an infinite rod (plate), without stress gradient along the surface (Moore and Evans [3]): one needs to measure the stresses on the vertical line above the point;

- for finite rod (plate) with stress gradient along the surface (this paper): one needs to measure the stresses and their gradient on the vertical line above the point;

- half-space without stress gradient along the surface (Ballard and Constantinescu, 1994 [2]): one needs to measure the stresses in the all the removed volume.

This reflects on the one hand the growing complexity of the problem and on the other hand the strength of the rod kinematics hypothesis. One should, however, remember that the rod solution is only an approximate solution, when compared to the fully three dimensional solution of the problem. In order to construct this solution one should dispose of the fundamental stress solution for a rectangular body of finite extension, which is not available for the moment.

\section{References}

[1] P. Ballard, M. Buliga, A. Constantinescu, Reconstruction d'un champ des contraintes résiduelles à partir des contraintes mesurées sur des surfaces succesives : Existence et unicité, C. R. Acad. Sci. Paris, série II 319 (1994) 1117-1122.

[2] P. Ballard, A. Constantinescu, On the inversion of subsurface residual stresses from surface stress measurements, J. Mech. Phys. Solids 42 (1994) 1767-1787. 
[3] M.G. Moore, W.P. Evans, Mathematical correction for stress in removed layers in X-ray diffraction residual stress analysis, SAE Trans. 66 (1958) 340-345.

[4] T. Nishimura, On axisymmetric residual stresses in tubes with longitudinally nonuniform stress distribution, ASME J. Appl. Mech. 60 (1993) 300-309.

[5] L. Trabucho, J.M. Viaño, Mathematical modelling of rods, in: G. Ciarlet, J.L. Lions (Eds.), Handbook of Numerical Analysis, vol. IV, Elsevier, 1996, pp. 487-650.

[6] B.Z. Vlassov, Pièces longues en voiles minces, Éditions Eyrolles, 1962. 